\title{
Towards space-grade 3D-printed, ALD-coated small satellite propulsion components for fluidics
}

\section{Kestilä, Antti}

2018-08

Kestilä , A , Nordling , K G M , Miikkulainen , V , Kaipio , M , Tikka , T , Salmi , M , Auer , A , Leskelä , M \& Ritala , M 2018 , ' Towards space-grade 3D-printed, ALD-coated small

satellite propulsion components for fluidics ' , Additive manufacturing , vol. 22 , pp. 31-37 . https://doi.org/10.1016/j.a

http://hdl.handle.net/10138/314395

https://doi.org/10.1016/j.addma.2018.04.023

cc_by_nc_nd

acceptedVersion

Downloaded from Helda, University of Helsinki institutional repository.

This is an electronic reprint of the original article.

This reprint may differ from the original in pagination and typographic detail.

Please cite the original version. 


\section{Accepted Manuscript}

Title: Towards space-grade 3D-printed, ALD-coated small satellite propulsion components for fluidics

Author: Antti Kestilä Kalle Nordling Ville Miikkulainen

Mikko Kaipio Tuomas Tikka Mika Salmi Aleksi Auer Markku

Leskelä Mikko Ritala

PII:

S2214-8604(16)30373-6

DOI: https://doi.org/doi:10.1016/j.addma.2018.04.023

Reference:

ADDMA 350

To appear in:

Received date: $\quad 30-12-2016$

Revised date: $\quad 18-4-2018$

Accepted date: $\quad$ 20-4-2018

Please cite this article as: Antti Kestilä, Kalle Nordling, Ville Miikkulainen, Mikko Kaipio, Tuomas Tikka, Mika Salmi, Aleksi Auer, Markku Leskelä, Mikko Ritala, Towards space-grade 3D-printed, ALD-coated small satellite propulsion components for fluidics, <![CDATA[Additive Manufacturing]]> (2018), https://doi.org/10.1016/j.addma.2018.04.023

This is a PDF file of an unedited manuscript that has been accepted for publication. As a service to our customers we are providing this early version of the manuscript. The manuscript will undergo copyediting, typesetting, and review of the resulting proof before it is published in its final form. Please note that during the production process errors may be discovered which could affect the content, and all legal disclaimers that apply to the journal pertain. 


\title{
Towards space-grade 3D-printed, ALD-coated small satellite propulsion components for fluidics
}

\author{
Antti Kestiläa,*, Kalle Nordling ${ }^{\mathrm{d}}$, Ville Miikkulainen ${ }^{\mathrm{b}}$, Mikko Kaipio ${ }^{\mathrm{b}}$, \\ Tuomas Tikka ${ }^{a}$, Mika Salmi ${ }^{\mathrm{a}}$, Aleksi Auer ${ }^{\mathrm{e}}$, Markku Leskeläb, Mikko \\ Ritala ${ }^{b}$ \\ ${ }^{a}$ Aalto University, School of Electrical Engineering, Department of Radio Science and \\ Engineering, P.O. Box 13000, FI 00076, Finland \\ ${ }^{b}$ Department of Chemistry, University of Helsinki, P.O. Box 55, FI 00014 Helsinki, \\ Finland \\ ${ }^{c}$ Aalto University, School of Engineering, Department of Mechanical Engineering, P.O. \\ BOX 14400, FI 00076, Finland \\ ${ }^{d}$ Finnish Meteorological Institute, P.O. BOX 503, FI 00101, Finland \\ ${ }^{e}$ House of Wisdom, Vanha Talvitie 11 FI 00580, Finland
}

\begin{abstract}
Space technology has been an early adopter of additive manufacturing (AM) as a way of quickly producing relatively complex systems and components that would otherwise require expensive and custom design and production. Space as an environment and long-term survivability pose challenges to materials used in AM and these challenges need to be addressed. Atomic layer deposition (ALD) is an effective coating method enabling conformal and precise coating of the complete AM print. This work analyses how an ALD coating of aluminium oxide on acrylonitrile butadiene styrene (ABS) and polyamide PA 2200 plastic AM prints benefits and protects them. This was studied in the context of in-space propulsion fluidics, where propellant flow properties also matter. AM was performed with material extrusion and selective laser sintering methods that are commonly used. Tests were performed with a simple bang-bang controller test setup and a mass spectrometer, and the existence of the coating was confirmed with scanning electron microscope imaging.
\end{abstract}

\footnotetext{
${ }^{*}$ Corresponding author.

Email address: antti.kestila@aalto.fi (Antti Kestilä)
} 
Keywords: space Technology, atomic layer deposition, Satellites, additive manufacturing, propulsion

2

\section{Introduction}

The rapid growth of small and micro-scale satellite utilization in a variety of areas has brought up the need for fast and cheap manufacturing processes for these satellite types [1]. As the volume of a satellite cannot be decreased indefinitely without losing functionality, a different approach is needed to retain that desired functionality, ideally without losing in turn in required manufacturing time, reliability and project costs.

Propulsion is an ideal test case for such an approach in manufacturing. More complex microsatellite missions often have a need for propulsion, and this subsystem requires components which have a complex structure and even some functionality in the structure itself. One reason for the poor performance of small satellite propulsion systems is the challenges caused by the satellite's small size. The propulsion system needs to pack the same functionality into a smaller space, while the providing significant $\Delta \mathrm{V}$. The system would thus benefit from mass optimization [2]. One demanding component in satellite propulsion is the often-used fluidics flow restrictor which is needed for stabilizing the gas flow to the thruster.

A promising method for mass optimization, and more generally manufacturing satellite systems and their support structures is additive manufacturing (AM) [3]. AM has already been used in existing or planned missions (see e.g. [4][5][6], and even in rocket engine designs [7]). AM is enabling a range of components and structures in the spacecraft not previously possible to manufacture [8][9]. However, metal AM-methods used for space applications such as laser sintering (see e.g. [10] for one case example) are currently not cost effective and the material and manufacturing costs associated are still high. Bringing down cost and component weight through the use of plastics could thus enable more effective use of 3D-printed technology in space applications.

Plastics, however, have disadvantages in a space environment, most critically degradation caused by UV radiation and outgassing [11]. For this reason plastic AM-manufactured components are not readily applicable in space without careful consideration of the type of material used for the component in question. 
In addition to relying on material choices when trying to avoid problems caused by the environment in space, another approach would be the use of a suitable coating to protect the AM-components (and in some cases other parts of the system) from the hazards of the space environment. Atomic layer deposition (ALD) has the best layer thickness control and surface conformality among the thin film deposition methods. This conformality is also potentially beneficial for AM technology as it could ideally smoothen and fix the relatively porous and rough structure manufactured by the printer. ALD has also a considerable variety of coating materials available [12].

ALD is a chemical vapor deposition method based on saturating and separating gas-solid reactions of two or more reactants which are repeated in a cyclic manner. The ALD cycle starts with a pulse from the first reactant that reacts with the solid surface. After the reaction has reached saturation, the gas phase is purged from the reactant surplus and gaseous side products. Next, the second reactant is pulsed to react with the solid surface. Again, a purge follows to remove the remaining reactant and gaseous side products. These cycles are repeated until the desired film thickness is deposited. Due to the surface-controlled, cyclic character of ALD, the thickness of films can be accurately controlled and highly conformal films can be deposited even on complex features.

In this work we study a manufacturing method which combines plastic 3Dprinting and ALD-coating to produce a propulsion component with the aim of improving its structural integrity, propellant flow performance, outgassing properties, and thermal resistance. The performance of a complex structure flow restrictor component is tested in an argon flow fluidics system originally designed for electric propulsion tests. Two different plastics are coated with ALD and tested. The plastics chosen are the most widely used plastic in 3D-printing acrylonitrile butadiene styrene (ABS), as well as the polyamide PA 2200. These two plastics are printed using different printing technologies - firstly, ABS by material extrusion (ME) and secondly PA 2200 with selective laser sintering (SLS). Other AM materials such as Stratasys's Verowhite and 3DSystem's Visijet PXL (dipped with Colorbond hardener) were also considered. However, Verowhite turned out to have a glass transition temperature which is too low for ALD with aluminum oxide and it started to outgas already at $50 \operatorname{deg} C$, while the Visijet PXL contained plaster which started to release water under a vacuum without any heating, prohibiting ALD. Aluminium oxide was used as the coating material as its ALD process is well understood and robust, and can be deposited at a low enough temper- 
ature required by the selected AM materials [13]. It also has been shown to provide an effective gas diffusion barrier for temperature-sensitive polymeric materials such LDPE [14]. While AM material temperature tolerances vary significantly depending on chosen material, generally they are lower than typical ALD process temperatures limiting the available coating materials. Aluminum oxide has been deposited at close to room temperature [15].

Without protective measures, space causes degradation in plastic components in several ways, such as reaction with atomic oxygen, UV light, ionizing radiation, vacuum and particles such as debris and micrometeoroids[16]. The component has to normally endure also large temperature fluctuations during an orbit in space. Atomic oxygen and high vacuum are the main degrading factors for a propulsion component which is by default protected against most of the above-mentioned environmental hazards, as it is placed inside the spacecraft. ALD aluminum oxide and titanium oxide have been already reported as effective protective coatings against atomic oxygen and UV light, respectively [11]. In the present work the focus was on the high vacuum of the space and the resulting outgassing that can for example dilute or contaminate the propellant.

\section{Methods and setup}

A restrictor with an ideal venturi channel geometry in a compact form was designed and printed from ABS as well as PA 2200. This way the mass flow choking phenomenon, typical of a venturi-type channel, could be tested and the results compared for ABS and PA 2200 materials and their underlying printing technologies.

The restrictor was included as a part of a bang-bang controller, as this controller type represents the simplest form of controllable in-space propulsion system. Thus the restrictor was tested in conditions similar to an actual use-case in space.

The theoretical mass flow of the restrictor was calculated using test setup data from the restrictor inlet pressure and compared with actual measured mass flow, for a range of aluminum oxide thicknesses. Smaller ABS plastic test pieces coated with the same layer thicknesses were also tested for outgassing intensity at a given temperature using a mass spectrometer. The coated restrictors were also imaged with a scanning electron microscope (SEM). 


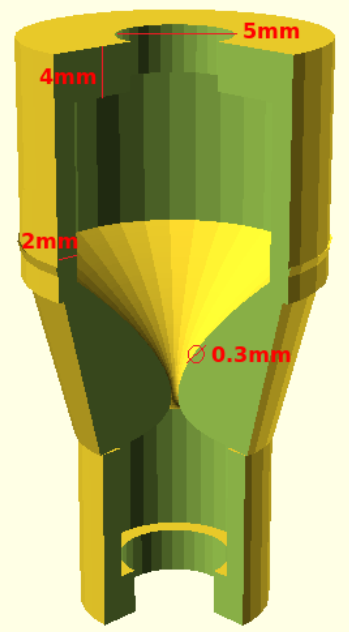

Figure 1: Dimensions of the designed venturi restrictor. Dimensions were confirmed with measurement.

\subsection{Restrictor design}

The venturi restrictor (hereon called just restrictor) was designed and manufactured so that the flow would choke in its throat section. The ready venturi restrictor cross-section from its CAD model can be seen in Figure 1 with critical dimensions. In order to obtain optimal channel shape for the venturi restrictor, classical theory of critical flow was used. Dimensions of the restrictor (cf. Figure 1) were defined using the area - Mach number ratio equation [17],

$$
\frac{A}{A^{*}}=\left(\frac{\gamma+1}{2}\right)^{-\frac{\gamma+1}{2(\gamma-1)}} \frac{\left(1+\frac{\gamma-1}{2} M^{2}\right)^{-\frac{\gamma+1}{2(\gamma-1)}}}{M}
$$

Where $A$ and $A^{*}$ are the cross-section area and critical cross-section area of the throat, $M$ mach velocity and gamma the ratio of specific heats for argon gas at room temperature. The output of this equation was used to define each area cross-section of the 3D-printed restrictor so that a consistent development of the flow's Mach-velocity starting from the inlet could induce sonic conditions in the venturi throat, and thus a critical mass flow.

\subsection{Setup and equipment}

For the ABS-made restrictors, the 3D-printer used was a Stratasys uPrint SE Plus, while the laser sintering EOS EOSINT P395 was used for the 
PA 2200 ones. The CAD-model was designed with Openscad. The program controlling the printer and converting the designed CAD-model into G-code was CatalystEX 4.4 software (Stratasys Inc., US) for material extrusion and the process software (PSW) 3.7 (EOS GmbH Electro Optical Systems, Germany) for powder-bed fusion (Selective Laser sintering).The AM-restrictor was printed with solid infill, so that all of its structure was filled in, intentionally leaving no empty spaces.

ALD of aluminum oxide was done with Picosun R-150 reactor using trimethylaluminum (TMA, Chemtura Manufacturing UK) and deionized water at a deposition temperature of $80{ }^{\circ} \mathrm{C}$ which is below the glass transition temperature of both ABS and PA 2200. Both reactants were evaporated from an external vessel held at room temperature and pulsed with pneumatic valves. The pressure inside the reactor was in the order of 5 mbar, maintained with a vacuum pump and a constant flow of nitrogen $\left(99.999 \% \mathrm{~N}_{2}\right)$ The deposition sequence was a $0.8 \mathrm{~s}$ pulse of TMA, a $5 \mathrm{~s}$ purge, a $0.8 \mathrm{~s}$ pulse of water, and again a $5 \mathrm{~s}$ purge. As aluminum oxide film thickness is complicated to measure directly on the printed plastic surface, it was measured by X-ray reflection on a piece of silicon cut from a $150-\mathrm{mm}$ Si(100) wafer, coated simultaneously with the printed plastics. The aluminum oxide film growth per ALD cycle was $0.88 \AA$ at these conditions and 228, 1140, and 2280 ALD cycles were used to deposit the 20,100, and 200-nm films. To confirm that aluminum oxide was deposited on the plastic components with high conformity, the inside cross-section of a venturi restrictor, coated with $200 \mathrm{~nm}$ of aluminum oxide and cut at the throat, was examined by a scanning electron microscopy (SEM).

In turn, the bang-bang controller tests on the restrictors were performed in a $10^{-6}$ mbar vacuum test chamber. The gas flowing through the test setup was a grade N35 purity argon gas, and the pressure as well as gas flow was controlled in several steps, see Figure 2 of the test setup used for the measurements.

\begin{tabular}{|l|l|l|l|l|l|l|}
\hline Material & $\begin{array}{l}\text { Tensile } \\
\text { strength } \\
\text { MPa }\end{array}$ & $\begin{array}{l}\text { Tensile } \\
\text { Modulus } \\
\text { MPa }\end{array}$ & $\begin{array}{l}\text { Strain at } \\
\text { break \% }\end{array}$ & 3D-printer & $\begin{array}{l}\text { Layer } \\
\text { thickness } \\
\text { mm }\end{array}$ & $\begin{array}{l}\text { Process } \\
\text { type }\end{array}$ \\
\hline ABS Plus & 33 & 2200 & 6 & $\begin{array}{l}\text { uPrint SE } \\
\text { Plus }\end{array}$ & 0.254 & $\begin{array}{l}\text { Material ex- } \\
\text { trusion }\end{array}$ \\
\hline PA 2200 & 48 & 1650 & 18 & $\begin{array}{l}\text { EOSINT } \\
\text { P395 }\end{array}$ & 0.1 & $\begin{array}{l}\text { Powder bed } \\
\text { fusion }\end{array}$ \\
\hline
\end{tabular}

Table 1: Sample material properties and their printer details. 


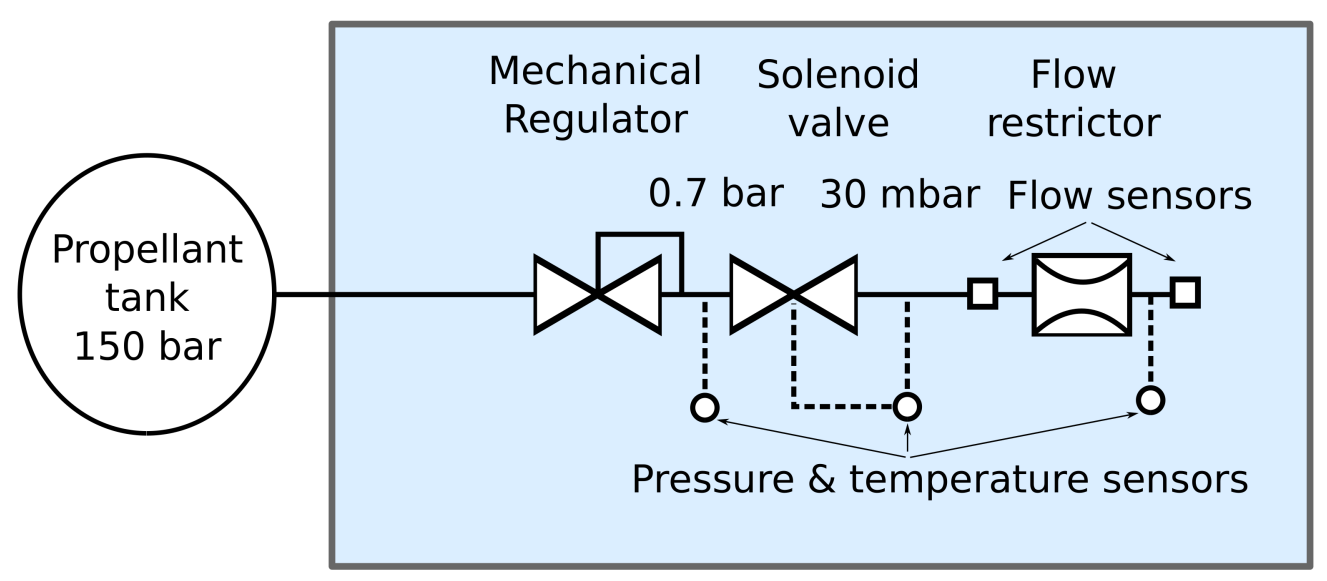

Figure 2: An overview diagram of the test setup used for measuring the restrictor performance. Besides the pressure argon tank, the rest of the setup was in a vacuum chamber.

The main pressure drop was performed using a mechanical two-stage regulator capable of withstanding high inlet pressures up to 100 bars. After that a high-speed solenoid valve provided flow control, and each in-between gas chamber in the setup contained a pressure and a temperature sensor, completing the control feedback loop. The mass flow measurements were performed with OMRON sensors tuned for low volumetric flows. The pressure and temperature were also measured immediately before and after the restrictor. The mass flow was calculated using the temperature measurements performed at the same location in the flow where also the pressure measurement was measured, calculating the density of the gas with these and converting the volumetric flow measured into mass flow. The restrictor had an OMRON sensor both before and after the printed flow restrictor.

Mass spectrometry on the ALD-coated pieces was performed by printing separate test pieces capable of fitting into the measurement chamber of the instrument, and were coated alongside the restrictors themselves in the same ALD run. Their outgassing tests were performed in a tubular steel vacuum chamber which was kept in vacuum using a rotary vane pump and a turbomolecular pump. The temperature in the chamber was monitored with an internal thermocouple, and the heating was performed using an external heat gun. The pressure inside the test system was in the order of $10^{-7}$ mbar. Outgassing species were monitored using a Hiden $\mathrm{HAL} / 3 \mathrm{~F} 501 \mathrm{RC}$ quadrupole mass spectrometer (QMS) with a Faraday detector, using an ionization energy of $70 \mathrm{eV}$.

SEM imaging on the test pieces was performed with a Zeiss Sigma VP (Schottky FEG emitter) in Aalto University's nanomicroscopy center, and relatively low level acceleration voltage of $1-1.5 \mathrm{kV}$ was used so as not to damage the 
plastic samples - though it was observed during the imaging that the ALD coating seemed to protect the sample better the thicker it was.

\section{Results and discussion}

\subsection{Measured spectrometry for outgassing}

Outgassing tests were performed on uncoated ABS samples, as well as on samples coated with 20, 100, and $200 \mathrm{~nm}$ of ALD aluminum oxide. PA 2200 was not included in the mass spectrometry results, as no discernible signal was detected from it when heating it during the measurements. The fractions that were measured with QMS were unique to the polymer and thereby their outgassing from the steel test chamber can be considered negligible. In the QMS measurements, the mass-to-charge ratio (m/z) of 102 (stemming most likely from styrene, which has an atomic mass of 104) was the one most easily discernible from the background, and its intensity was therefore used in quantifying the outgassing behavior of the samples at each temperature.[18][19] show previously performed similar tests where ABS styrene was successfully detected. Figure 3 portrays the intensity of the $\mathrm{m} / \mathrm{z}=102$ signal as a function of sample temperature. The difference in the outgassing from samples with ALD aluminum oxide as compared to uncoated samples was mostly negligible. Two samples coated with $100 \mathrm{~nm}$ and $200 \mathrm{~nm}$ of aluminum oxide seemed to show less outgassing than other samples. However, this difference might not be due to the coating itself, but can stem from measurement uncertainty in the experimental setup. The temperature threshold for significant outgassing seems to be at around $90{ }^{\circ} \mathrm{C}$, which is about fifteen degrees lower than the glass transition temperature of ABS. In any case, the ALD process has no detrimental effect on the outgassing properties of the ABS samples, although it does not improve these properties either. The reason for this is not entirely clear. In principle, outgassing channels through the aluminum oxide films could be created by a thermal deformation of the ABS samples, or by the internal pressure created by the outgassing species. Unfortunately, it is difficult to estimate both the pressure of the gases formed, and the effects of pressure on the coatings, especially when the aluminum oxide films have nanoscale thicknesses.

Previous outgassing tests performed by Minton et al. [11] indicated that thin ALD aluminum oxide layers significantly reduce the vacuum ultraviolet light induced outgassing of poly(methyl methacrylate). Light irradiation, however, probably affects primarily the surface of an irradiated sample, whereas 
heating creates outgassing throughout the entire bulk of a heated sample, making it not possible to compare the two different outgassing mechanisms. Temperature induced outgassing behavior should therefore be studied further, in order to find thin film materials which are sufficient for blocking most or all of the outgassed species.

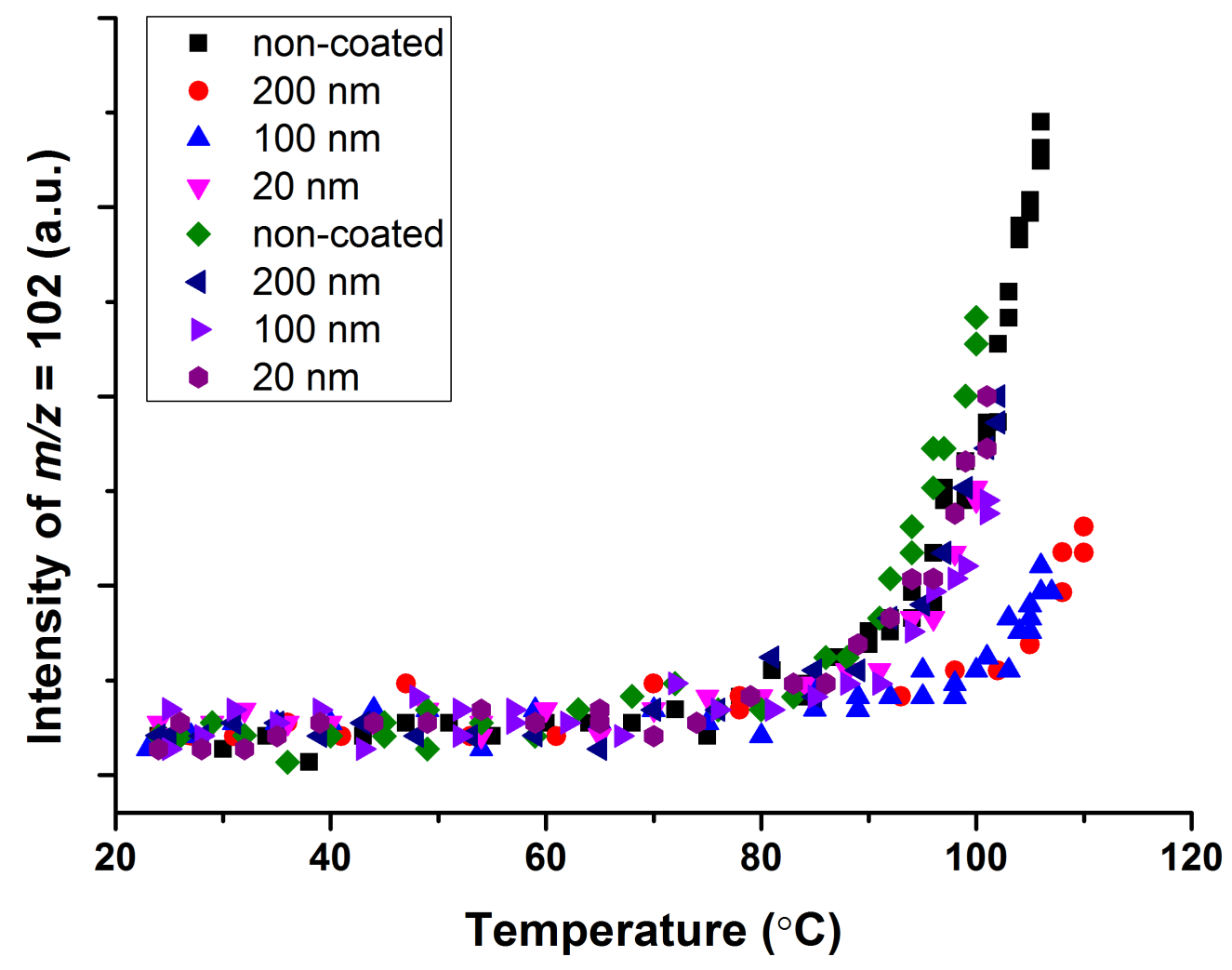

Figure 3: Intensity of the QMS $\mathrm{m} / \mathrm{z}=102$ a.u. signal as a function of temperature. Signals from eight separate ABS+ samples were measured: two non-coated samples, two samples coated with $20 \mathrm{~nm}$, two samples coated with $100 \mathrm{~nm}$, and two samples coated with 200 $\mathrm{nm}$ of aluminum oxide.

\subsection{Measured structural integrity and mass flow properties}

The restrictors with the range of coating thicknesses (ABS+ and PA 2200

for $0,20,100$ and $200 \mathrm{~nm}$ ) were also tested for their flow properties in a vacuum 
test setup with a bang-bang controller, mimicking the simple flow control system found in many propulsion systems in space. As the setup does not have other flow-modifying components, and the way how the restrictor affects flow is well known in theory, by comparing the theoretical and measured mass flow the efficiency of the coated AM restrictors can be evaluated. The pressure at the inlet of the restrictor was measured, and with this value the compared theoretical mass flow was calculated. The solenoid valve in the setup was opened until the pressure at the restrictor inlet increased above 20 millibars, after which the valve was closed and the argon in the inlet was allowed to discharge through the restrictor. Once the inlet pressure decreased below 2 millibars, the solenoid valve was again opened for 150 milliseconds, filling the inlet chamber and the cycle repeated, resulting in a pulsing profile. The restrictor test for each coating thickness lasted about 30 minutes with hundreds of argon pulses going through the restrictor. The mass flow both before and after the restrictor was measured, as well as the inlet and outlet pressure and temperature. The test chamber pressure was measured to be a high vacuum of around $10^{-6}$ mbar before the start of the test. The chamber and test setup temperature was a steady $32.5 \mathrm{deg} C$, with a maximum of half a degree Celsius change measured during the whole test.

Using conservation of mass in a flow, the isentropic flow relations and the equation of state gives an ideal mass flow (derived from the basic isentropic flow relations, shown for example in [20], and the equation of state),

$$
\dot{m}=\frac{A P_{t}}{\sqrt{T_{t}}} \sqrt{\frac{\gamma}{R_{s}}}\left(1+\frac{\gamma-1}{2} M^{2}\right)^{\frac{\gamma+1}{2(\gamma-1)}}
$$

where $\mathrm{A}$ is now the restrictor throat cross-section, $R_{s}$ the gas constant for argon, $P_{t}$ and $T_{t}$ the total inlet pressure and temperatures, respectively, $\gamma$ is again the ratio of specific heats for argon gas, and $M$ still the Mach velocity of the gas. As sonic conditions occur in the throat of the restrictor and so M $=1$ there, the equation gives the value of a choked flow, restricting mass flow to a constant value after the throat (for a specific inlet pressure). Equation 2 gives an ideal baseline with which to compare the measured mass flow going through the restrictor.

Figure 4 shows the resulting ideal and measured mass flows and the difference between them, for both materials. This difference is assumed to indicate the efficiency of the restrictor compared to an ideal restrictor, so for example how accurately the restrictor has been manufactured, does it have surface defects that affect the flow, or is it porous due to possible micro-level (and 
smaller) manufacturing errors. A positive value in the Figure plots indicates a worse than ideal mass flow value, pointing to inefficiency, while a negative value represents a larger than ideal mass flow, which in turn means the gas is escaping from the restrictor due to leaks in the printed structure.

The assumption of isentropic flow is used when calculating the ideal flow as well as in the design of the restrictor. No heat transfer occurred as the restrictor was in the vacuum of the test chamber. Also, the measured temperature stayed stable to within half a degree Celsius throughout the entire test. Increased friction due to very small defects and deformities in the structure is possible considering that the actual inner shape of the restrictor is not perfectly manufactured, but the overall effect of such defects should be minor. The results in Figure 4 seem to indicate that the coating affects the efficiency of the flow through the restrictors.

Further on, by repeating these tests several times on the same uncoated restrictor, the restrictor efficiency changed with every test, the difference between ideal and measured mass flows going more into the negative each time. This means there is increasingly more mass flow in than there should be ideally, and is possibly caused by the restrictor sample effectively breaking down slightly more with every trial. In terms of Equation 2, it would be as if the cross-sectional area of the throat would increase more than the decrease in measured total pressure. Thus Figure 5 seems to indicate that the coating strengthens the mechanical structure of the ABS restrictor. The same was not observed with the coated ABS restrictors even after more test runs than those done with the uncoated restrictor, and neither with any of the PA 2200 restrictors, uncoated or coated.

The surface of both ABS plus and PA 2200 restrictors, as well as the part of the ABS throat cross-section was SEM imaged to determine how well the coating adhered to it, the results of which can be seen in Figure 6 . The surface (Figure 6 A and B) of the PA 2200 restrictors was observed to smoothen out with increased coating thickness, which is the most likely explanation as to why the flow through them improved. Notice that even the thickest coating was 0.2 microns, and so cannot alone explain the dramatically increased smoothness seen in the images. Possibly, the surface smoothing is a combined thermal and chemical effect: at ALD temperature, PA 2200 surface softens and/or reacts with TMA. Energy-dispersive X-ray spectroscopy (EDX) analysis confirmed that the coating had indeed formed over the piece.

As an example of the ABS-made restrictors, Figure 6 (C and D) shows a 


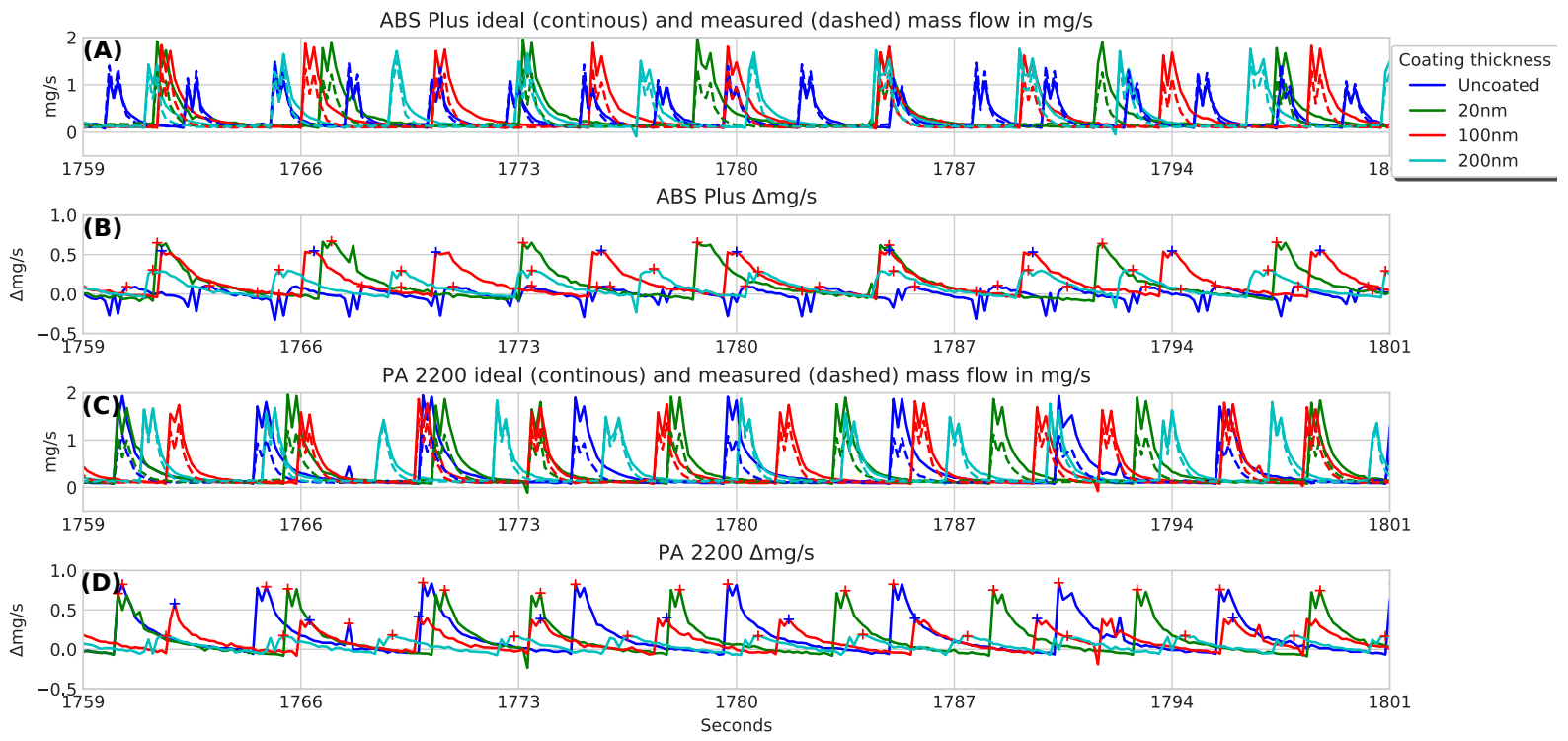

Figure 4: The ideal and measured mass flow going through four different coated restrictors in a bang-bang controller for both ABS plus and PA 2200 materials can be seen in plots (A) and (C). In turn, plots (B) and (D) represent the difference between the ideal and measured mass flow of these same plots, and show more clearly the observed change due to coating thickness. (B) For restrictors ranging from uncoated to 20, 100 and $200 \mathrm{~nm}$ of aluminum oxide coating, the difference steadily decreases as the thickness of the coating increases. A positive mass flow value likely indicates inefficiency. The blue plot (uncoated) veers quite a lot to the negative side, likely indicating the uncoated restrictor sample is leaking. In plot (D) a more rapid decrease in the mass flow difference occurs with restrictors made out of PA 2200, but does not go into the negative side.

cross-section at the restrictor throat, where the interface of the 200nm ALDcoating can be seen (the restrictor was broken in half at the throat so the image could be taken), while image $\mathrm{E}$ is taken from another location on the same cross-section. C,D and E show how the coating has formed well and smoothly, and is broken only by external damage (such as opening the restrictor as was done with these images). The same good coating quality was observed in all the ABS restrictors.

As a sidenote, during SEM imaging it was noticed that increased coating improved the quality and success of the images. This was most likely due to the coating protecting the plastic from the electron beam that otherwise would melt it, as was the case with the baseline uncoated restrictors. 

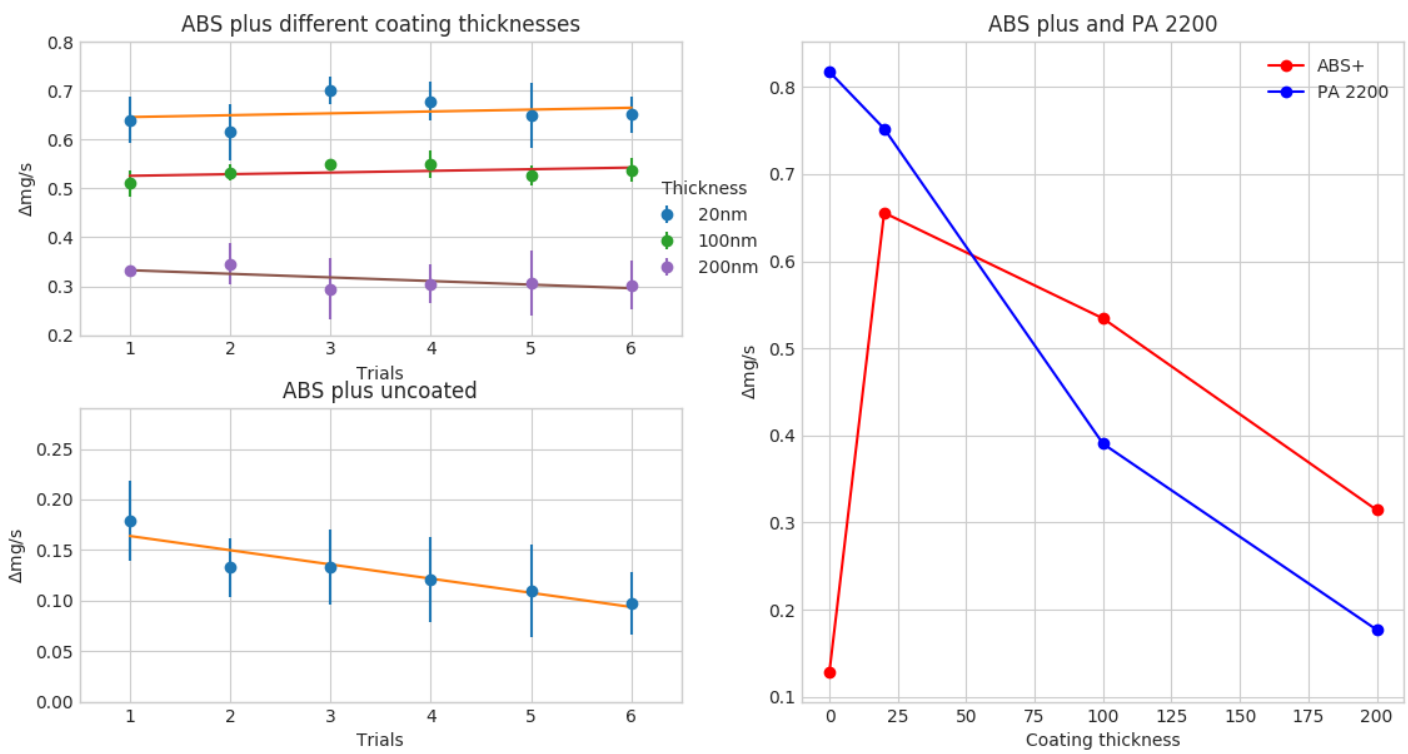

Figure 5: Results of the tests where all restrictors were placed in about 30 minutes (several hundred pulses) long series of trials, and the mean of all the pulses in each trial was taken. (A) Mean pulse amplitudes per trial, along with their standard deviations, for all coated restrictors showed no decrease, while (B) the uncoated restrictor showed a straightforward drop for each trial, indicating the printed structure breaking down a little every trial. This was observed only in the uncoated ABS-material restrictor, but not the uncoated PA 2200 made prints. (C) in turn shows the "mean of means" of plots (A) and (B) in order to bring out how these mean pulse amplitudes vary with respect to coating thickness for both materials. A decrease in mean amplitude is observed in both cases as the coating thickness increases, with the notable exception of the uncoated ABS restrictor.

Several measurement sensors for pressure, temperature and mass flow were used (see Figure 2) in order to increase redundancy in the setup. In order to lessen measurement uncertainty, Figure $5 \mathrm{~A}$ and B plots show the pulse mean amplitude (and its standard deviation) of 30-minute long series of pulses, while plot $\mathrm{C}$ in turn shows their mean to better illustrate the point.

\subsection{Measurement uncertainty}

The biggest uncertainty in the QMS measurements (section 3.1) was most likely caused if one sample was heated unevenly compared to another. A heat gun was used in warming up the sample in the measurement chamber, and so might have warmed up the samples slightly differently from each each other and that of what was measured by the temperature sensor. If 


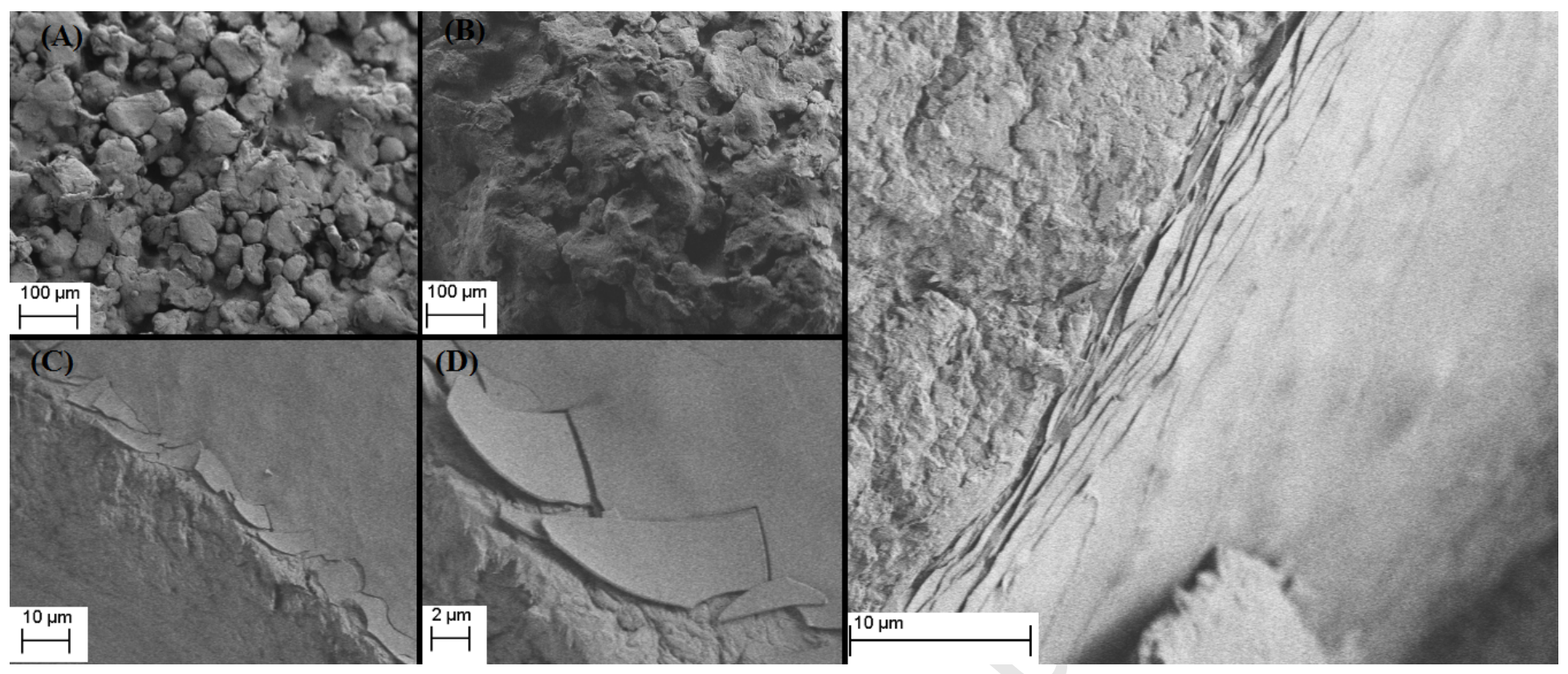

Figure 6: SEM images - (A) Uncoated polyamide print compared to (B) $200 \mathrm{~nm}$ coated one. The different surface roughness is the most likely reason from improved flow properties, and is possibly due to the ALD process reacting with escaping water. (C) A close up of the ALD coating breakline at an ABS print cross-section (D) and a even closer look at broken and dislodged individual ALD coating pieces.(E) Another example of a print cross-section where the ABS plastic-aluminium oxide interface is clearly shown.

there was outgassing from any possible contamination inside of the chamber, its magnitude would be insignificant compared to the outgassing from the relatively large polymer block itself.

As for uncertainty in mass flow measurements (section 3.2), both mass flow and pressure sensors have around 0.5 percent accuracy of their full scale and no detectable drift (the measured pulses were around 20 mbar, which is well within the capabilities of the sensors). After vacuum formation, the test chamber vacuum stayed relatively stable as can be seen in Figure 4 measurements. Only measurements performed after the chamber calmed down were used. Several long runs of mass flow and pressure measurements were performed in order to counter any random errors in the pressure and mass flow sensors. A maximum of half a degree change in temperature in the gas running through the mass flow measurement setup was recorded.

\section{Conclusions}

In conclusion, the present study revealed interesting benefits in combining $\mathrm{AM}$ and ALD. The aluminum oxide coating, even as thin as 20 nanometers, improved the structural integrity for the ABS plus restrictors and progressively smoothed out the PA surface improving the argon flow through the 
restrictor. Based on results shown in Figures 4,5 and 6, the improved flow effect observed in the ABS restrictor samples might be due to increased surface smoothness, although based on SEM visual inspection the uncoated sample didn't look to have a particularly rougher surface than the coated ones. The flow differences between the uncoated and coated samples might in turn be due to the sealing up of micro and nano-level cracks in the print by the coating. Also, as the thickness of the coating increases it might patch up larger cracks. The ALD coating thus might be able correct some of the minute flaws and imperfections in the ME AM process.

The tests on the ABS prints also gave some indication that the coating used in this work might slightly mitigate outgassing at higher temperatures, but overall this remains inconclusive, and requires more research. Such research includes a more detailed investigation of which mechanisms of outgassing (e.g. sample heating, or induced by external sources) can be most effectively prevented with ALD coating, as well as through selection of more suitable materials for both ALD and AM.

Other interesting future research directions include testing how a combination of ALD and AM withstands mechanical forces (in a space context for instance launch forces and the structural vibrations they cause), testing new coating materials and especially their layered combinations for useful properties such as improved radiation protection, as well as using ALD with metal AM. Temperature during the coating process is important in terms of the AM component survivability as well as in terms of what coating materials can be used. Thus during future work it is also good to try to understand how to increase the temperature tolerance of the AM material so as to be able to coat it with a wider selection of materials that require higher temperatures.

Better use of AM in space could reduce significantly the time taken in manufacturing many space systems as well as the cost of manufacture. Additionally, AM could help to build space systems not possible using traditional manufacturing methods. The choice of a suitable coating material could also protect a propulsion system from more aggressive propellants that would otherwise cause damage to its components, especially if they are made using materials usable in AM. Other use cases could, for instance, include creating safer medical applications by better isolating the AM component from the human body.

As AM develops further into a more precise manufacturing method, combining the excellent conformity of atomic layer deposition, a wide variety of 
possible coating materials and the possibility of multiple different coating layers could enable the manufacture of complex and very small-scale technology. Further on in the future, a combination of these two methods in a single manufacturing process, and even into a single machine, where AM and ALD are used interchangeably layer by layer, could enable completely new applications.

\section{Acknowlegments}

The study would like to thank Professor Esa Kallio from Aalto University School of Electrical Engineering for his financial support of this work. The work has been supported by Finnish Centre of Excellence in Atomic Layer Deposition (Academy of Finland, project no. 284623), The Finnish Academy of Science and Letters, The Finnish Society of Sciences and Letters, and the Finnish Cultural Foundation.

\section{References}

[1] R. Sandau, Status and trends of small satellite missions for Earth observation, Acta Astronautica 66 (2010).

[2] V. Zakirov, L. Luming, Propulsion challenges for small spacecraft: 2005, Tsinghua Science and Technology 11 (2005) 507-514.

[3] T. Mosher, B. Stucker, Responsive space requires responsive manufacturing-part ii, in: AIAA Space 2004 Conference and Exhibit, San Diego, CA, US.

[4] G. Moore, W. Holemans, A. Huang, J. Lee, M. McMullen, J. White, R. Twiggs, B. Malphrus, N. Fite, D. Klumpar, et al., 3d printing and mems propulsion for the rampart $2 \mathrm{u}$ cubesat, in: 24th Annual AIAA/USU Conference on Small Satellites 2010, Logan UT, US.

[5] T. Stevenson, E. Imken, E. G. Lightsey, Design and testing of a cold gas thruster for an interplanetary cubesat mission, Acta Astronautica 4 (2015) 371-386.

[6] C. Auletti, Icesat-2: A mission of firsts, NASA CuttingEdge Newsletter 12 (2016). 
[7] J. G. Misra, R. Carter, Additive manufacturing of aerospace propulsion components, https://ntrs.nasa.gov/search.jsp?R=2015002306, 2015. Presented at The Additive Manufacturing Conference, Pittsburgh, PA, October 1, 2015.

[8] D. Espalin, D. W. Muse, E. MacDonald, R. B. Wicker, 3d printing multifunctionality: structures with electronics, The International Journal of Advanced Manufacturing Technology 72 (2014) 952-978.

[9] J. A. V. Vilán, F. A. Agelet, M. L. Estévez, A. G. Muiño, Flight results: Reliability and lifetime of the polymeric 3d-printed antenna deployment mechanism installed on xatcobeo \& humsat-d, Acta Astronautica 107 (2015) 290-300.

[10] J. Quincieu, C. Robinson, B. Stucker, T. Mosher, Case study: selective laser sintering of the ususat ii small satellite structure, Assembly Automation 25 (2006) 267-272.

[11] T. K. Minton, B. Wu, J. Zhang, N. F. Lindholm, A. I. Abdulagatov, J. OPatchen, S. M. George, M. D. Groner, Protecting polymers in space with atomic layer deposition coatings, Applied Materials and Interfaces 2 (2010) 2515-2520.

[12] R. Johnson, S. B. Hultqvist, A brief review of atomic layer deposition: from fundamentals to applications, Materials Today 17 (2014) 236-246.

[13] V. Miikkulainen, M. Leskelä, M. Ritala, L. Puurunen, Crystallinity of inorganic films grown by atomic layer deposition: Overview and general trends, Journal of Applied Physics 113 (2013) 021301-021301-101.

[14] J. D. Ferguson, A. W. Weimer, S. M. George, Atomic layer deposition of al2o3 films on polyethylene particles, Chemistry of Materials 16 (2004) $5602-5609$.

[15] M. D. Groner, F. H. Fabrequette, J. W. Elam, S. M. George, Lowtemperature al2o3 atomic layer deposition, Chemistry of Materials 16 (2004) 639-645.

[16] E. Grossmann, G. I., Space environment effects on polymers in low earth orbit, Nuclear Instruments and Methods in Physics Research 208 (2003) 48-57. 
[17] J. Anderson, Fundamentals of aerodynamics, McGraw-Hill, 3rd edition, 2001, p. 567.

[18] K. J. Budde, W. J. Holzapfel, M. M. Beyer, Application of ion mobility spectrometry to semiconductor technology: Outgassings of advanced polymers under thermal stress, The electrochemical Society 142 (1995) 888-897.

[19] T. Streibel, R. Geißler, M. Saraji-Bozorgzad, M. Sklorz, E. Kaisersberger, T. Denner, R. Zimmermann, Evolved gas analysis (ega) in tg and dsc with single photon ionisation mass spectrometry (spi-ms): molecular organic signatures from pyrolysis of soft and hard wood, coal, crude oil and abs polymer, Journal of Thermal Analysis and Calorimetry 96 (2009) 795-804.

[20] J. Anderson, Fundamentals of aerodynamics, McGraw-Hill, 3rd edition, 2001. Chapter 7.2 .5 with "isentropic relations" and chapter 8.4 with "special forms of the energy equations". 